\title{
Effects of firn ice models on radio neutrino simulations using a RadioPropa ray tracer
}

\section{B. Oeyen, ${ }^{a, *}$ I. Plaisier, ${ }^{b}$ A. Nelles, ${ }^{b, c}$ C. Glaser $^{d}$ and T. Winchen ${ }^{e}$}

\author{
${ }^{a}$ Ghent University, Dept. of Physics and Astronomy \\ Proeftuinstraat 86, B-9000 Gent, Belgium \\ ${ }^{b}$ DESY, Platanenallee 6, D-15738 Zeuthen, Germany \\ ${ }^{c}$ Erlangen Center for Astroparticle Physics (ECAP), Friedrich-Alexander-University \\ Erlangen-Nuremberg, Erwin-Rommel-Str. 1, D-91058 Erlangen, Germany \\ ${ }^{d}$ Uppsala University, Dept. of Physics and Astronomy Angström Laboratory \\ Lägerhyddsvägen 1 ,Uppsala SE-752 37, Sweden \\ ${ }^{e}$ Vrije Universiteit Brussel, Astrophysical Institute \\ Pleinlaan 2, B-1050 Brussels, Belgium \\ E-mail: Bob.Oeyen@uGent.be, Ilse.Plaisier@desy.de, Anna.Nelles@desy.de, \\ Christian.Glaser@physics.uu.se, Tobias.Winchen@rwth-aachen.de
}

The simulations for in-ice radio detectors rely on analytically solvable exponential ice models, allowing for computationally fast ray tracing. However, these models do not encompass the whole complexity of the ice which may result in systematic errors on the simulations and reconstructions. We incorporated a numerical ray tracer module in NuRadioMC based on RadioPropa with acceptable speed and accuracy that focuses on accommodating more complex ice models. Presented here are the ray tracer's implementation, and a first simulation and reconstruction using this new module. This shows that changing the ice model from an exponential profile to a double-exponential profile (firn model) has non-trivial effects on the timing. As this is important for distance and direction reconstruction of neutrino events, we hope to investigate this influence further once other effects like in-ice reflections or birefringence are implemented in this ray tracer as well.

$37^{\text {th }}$ International Cosmic Ray Conference (ICRC 2021)

July 12th - 23rd, 2021

Online - Berlin, Germany

\footnotetext{
${ }^{*}$ Presenter
} 


\section{Introduction}

Simulations for in-ice radio detectors - a promising technique to detect neutrinos of energies beyond the ones measured thus far - highly depend on the ice modeling, and an accurate ray tracing is necessary. Currently, mainly exponential models of the ice are used in simulations as the ray paths can be analytically solved which allows for computationally fast ray tracing. Solutions for this model result in three types of rays: direct (depth decreasing monotonically along the path), refracted $^{1}$ (path shows a turning point) and reflected rays (reflection of the air-ice boundary), with a maximum of two solutions per pair of start- and endpoint. For Summit Station in Greenland, the location of the new Radio Neutrino Observatory - Greenland [1], this model $^{2}$ is described by:

$$
n=1.78-0.51 \cdot \exp (-z / 37.25 \mathrm{~m}) \quad z \leq 0 \mathrm{~m}
$$

with $n$ the refractive index and $z$ the $\mathrm{z}$-coordinate of the evaluated point in the local coordinate system $^{3}$. Although these models describe the refractive index of ice well to first order [3], they do not comprise the whole complexity of the ice such as in-ice reflection due to density discontinuities, or horizontal propagation through the firn. To improve the modeling in simulations and to eventually reduce uncertainties in the event reconstruction, a more precise calculation of the propagation of radio waves through ice is needed.

NuRadioMC [4] - the most recent software to simulated events in radio neutrino detectors - handles the signal generation and propagation completely separately, which makes it easy to add and swap out ray tracing modules to deal wit different kind of ice models. The ray tracer module propagates the ray from the neutrino interaction point (starting point) to an antenna of the detector (endpoint). To accommodate the use of more complex ice models, we incorporated RadioPropa [5], a numerical ray tracer, into NuRadioMC. This contribution will cover the implementation of the module, new features and their influence on neutrino simulations.

\section{Technical description}

RadioPropa is a modular numerical ray tracing code written in $\mathrm{C}++$ [5] which can be used freely to construct a simulation. The most important modules for our purpose are: Source, PropagationCK, IceModel, Observer, and Discontinuity (Figure 1). Source is the starting point (neutrino interaction vertex) of the ray tracing and specifies the location, direction and amplitude of the signal. Propagation $C K$ handles the propagation of a radio ray by calculating every distance step the bending of the path due to the change in refractive index, which is given for every point in space by the IceModel. Observer is a surface with a specified position and shape, which denotes the end of the ray tracing once the surface is reached. They primarily function as end points (detector antennas) but can also serve their purpose as boundary conditions; for example at the bottom of a glacier. Finally, Discontinuity is a surface that describes a non-continuous change in refractive index and hence produces both reflected and transmitted rays using Fresnel's law, for example at the ice-air boundary. Note that a discontinuity in the refractive index should be declared explicitly using this module.

\footnotetext{
${ }^{1}$ The refractive index changes with depth allowing for refracted rays. A derivation can be found in appendix C of [2] ${ }^{2}$ The model is obtained by a fit to data of represented in [2].

${ }^{3}$ The $\mathrm{x}$-axis points east and the $\mathrm{z}$-axis points vertically upwards at the sky with the origin at the surface.
} 


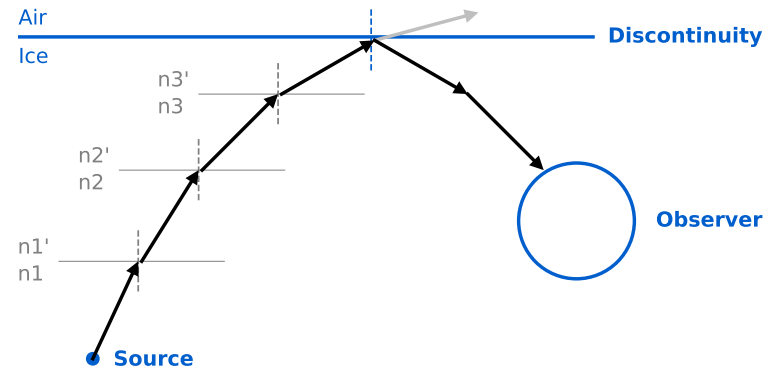

Figure 1: The RadioPropa ray tracers start at the source and employs small finite distance steps after which the classic law of Fresnel is evaluated to calculate the bending of electromagnetic rays until it reaches an observer. When reaching a discontinuity both reflected and refracted rays from this law are propagated further.

\subsection{Implementation in NuRadioMC}

A ray tracing module integrates RadioPropa into NuRadioMC by using an iterative method to find the ray tracing solutions. RadioPropa needs an initial launch direction for the signal ray at the starting position, after which the code propagates this ray further. Numerically searching for the right launch direction takes a lot of time because many rays need to be traced. However, smart geometric restrictions and an iterative method can limit the time needed.

The solutions are found by calculating rays in a specific range of directions of zenith $\theta$ and azimuth $\phi$ in the hope to hit the detector. Since it is impossible to numerically hit a infinitesimal point, the detector's antennas are represented by spherical observers. In order to make the solution accurate at both the launch and arrival of the signal, the size of the sphere and the angular step size need to be small. Unfortunately, since smaller steps result in more rays to trace, this increases the computational effort. Therefore, several measures are taken in order to reduce the computing time:

1. Any ice model can be implemented in RadioPropa, as long as it is defined at each point $(\mathrm{x}, \mathrm{y}, \mathrm{z})$. However, currently only ice models with a depth dependent refractive index are implemented, and thus there is no ray bending in the xy-plane. Therefore, the ray tracing can be performed in $2 \mathrm{D}$ by restricting the ray to a vertical plane holding the start and end point $\left(\phi=\phi_{\text {antenna }}\right)^{4}$.

2. Because the refractive index increases with depth, the rays will always be refracted downwards, never upwards. Therefore, rays shot at a zenith $\theta$ below the antenna's zenith $\theta_{\text {antenna }}$ will never reach that antenna, so this region can be excluded $\left(\theta \geq \theta_{\text {antenna }}\right)^{4}$. For ice models with a reflective layer below the start point this is not the case and other measures are taken.

3. The ray tracer scans the residual search region in successive steps where each step limits the search region further for the next iteration (Figure 2). In the first stage, accuracy is irrelevant and the tracer scans the region with big angular steps and a large spherical antenna. Only directions for which the rays are detected are saved and this new region will be scanned more accurately in the next iteration with a smaller sphere and smaller angular steps. This cycle repeats until the necessary accuracy is reached. In the final state there may still be a bundles of rays, in which case the ray pointing most towards the antenna is chosen as the solution.

\footnotetext{
${ }^{4} \theta_{\text {antenna }}$ and $\phi$ antenna are the zenith and azimuth of detector antenna seen from the neutrino in the global coordinate system.
} 


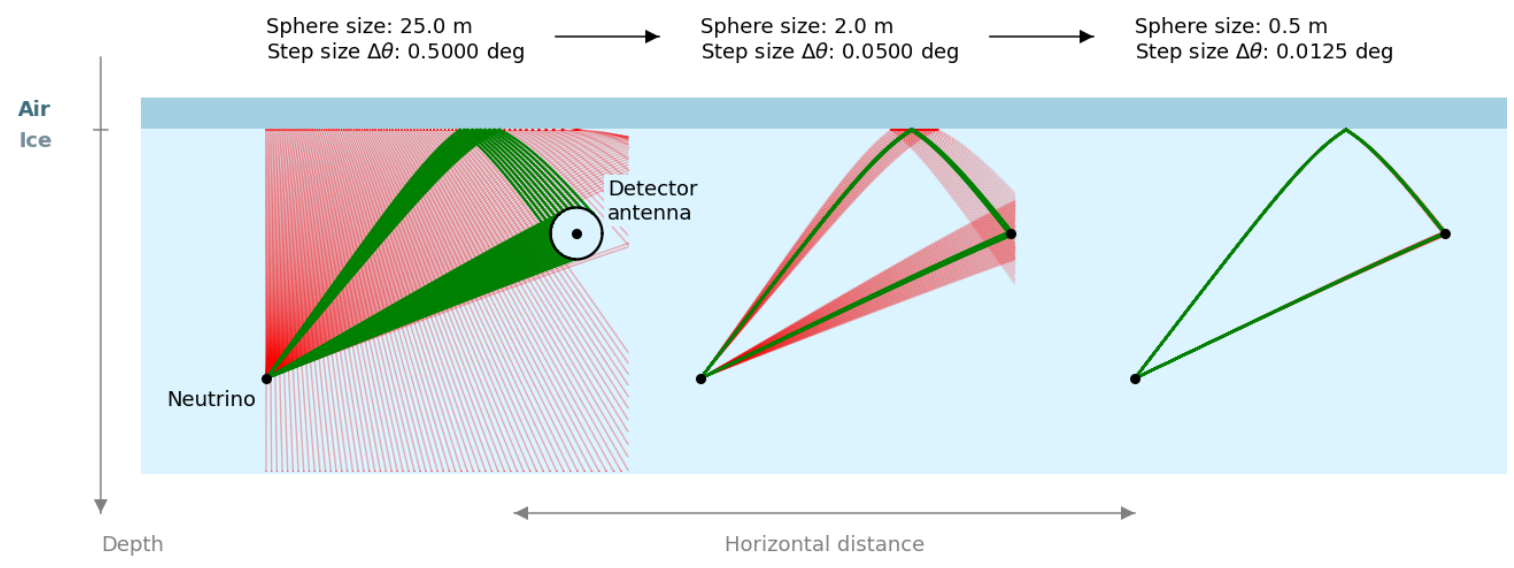

Figure 2: The iterative method of the RadioPropa ray tracer uses successive steps, resulting in more precise solutions of the ray paths between the neutrino event and the antenna. By default this method utilizes three steps with sphere size decreasing from $25 \mathrm{~m}$ to $2 \mathrm{~m}$ to $0.5 \mathrm{~m}$ and zenith step size $\Delta \theta$ decreasing from $0.5^{\circ}$ to $0.05^{\circ}$ to $0.005^{\circ}$.

\subsection{Accuracy of the RadioPropa ray tracer}

The accuracy of the solution is important for the simulation as they influence the specific signature of the detected signal used in reconstruction and analysis methods. A first crucial parameter is the viewing angle, which is the angle between launch and shower direction, since it determines the shape of the Askaryan radio signal. Other essential parameters are: arrival time, the signal arrival direction and the polarisation of the electric field. They are used for reconstructing the position and direction of the neutrino event and the signal direction.

For the RadioPropa ray tracer, the various signal parameters mentioned above are accurate when comparing its solutions to the solutions of the analytic ray tracer (Figure 3). The comparison was performed using the exponential ice model for Summit, Greenland (Equation 1), since the analytical ray tracer can only solve for these smooth kind of ice profiles. RadioPropa, taking approximately 50 times longer to compute, correctly found solutions for the exact same events as the analytical ray tracer, without missing or adding other solutions. The timing is accurate up to less then a nanosecond, which is required for a good timing between different signals. The viewing angle is precise to less then $0.02^{\circ}$ with the direct rays centered around a $0^{\circ}$ difference, and refracted and reflected rays shift to slightly higher angles of which the reflected the most pronounced. The arrival zeniths are all slightly bigger for RadioPropa with the same tendency in shift, according to the ray type. The polarisation, which is defined as $P=\arctan \left(\sqrt{E_{\phi}^{\text {fluence }} / E_{\theta}^{\text {fuence }}}\right)$, of the direct and refracted rays has a very high accuracy, whereas the reflected rays have a bigger spread. The differences between the RadioPropa tracer and the analytical tracer is the result of the limited numerical precision of the iterative step. For more accurate results both the step size and sphere can be further reduced in an additional iterative step. 


\section{Comparison of numerical ray tracer to analytical raytracer for:}
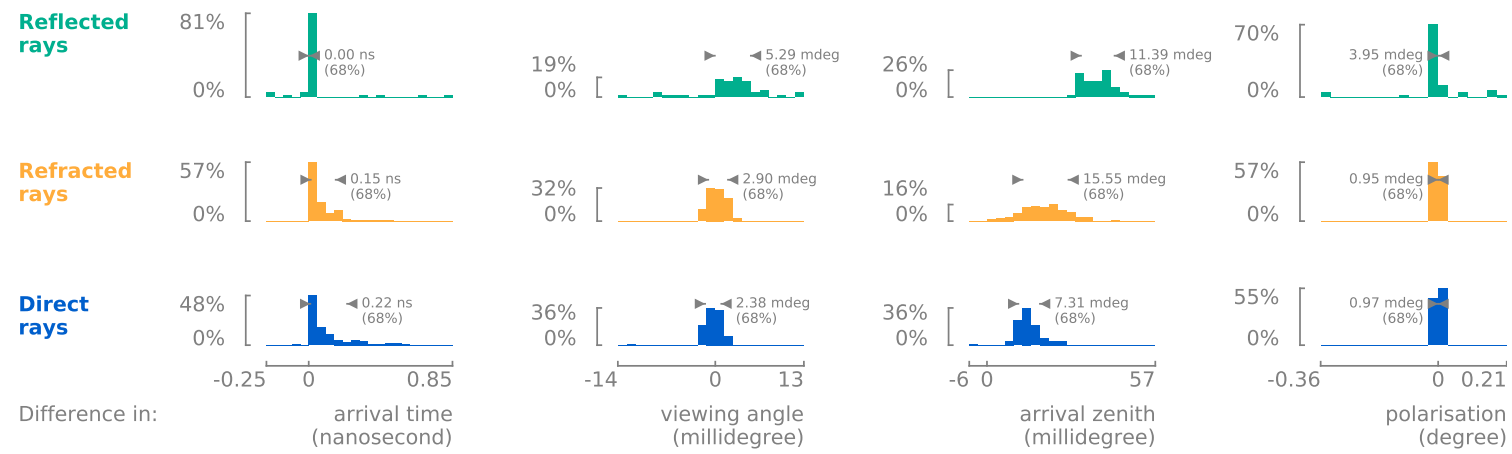

Figure 3: The RadioPropa ray tracer, compared to the analytic ray tracer, has a resolution with a peak value around zero for the arrival time, viewing angle and polarisation angle, and a peak value slightly shifted to higher values for the signal zenith angle. Furthermore, the peak and the spread of the peak (given by a $68 \%$ interval) may depend on the ray type for certain variables.

\section{Features in RadioPropa}

An initial pair of complex ice features is implemented and tested together with the RadioPropa module as this is the main motivation for its development. A first extension is the replacement of exponential ice models by a double exponential ice model, called the firn model, as suggested by [2]. Further, the observation of classically forbidden rays out of the shadow zone [3] inspired the incorporation of horizontal and surface wave modes. In the future we intend to expand the code further beyond these two features by adding effects like birefringence and internal reflective layers.

\subsection{Firn model}

The firn model describes the ice using two exponential profiles, one for the upper part of the ice and one for the lower bulk ice, which are then connected to each other at a critical depth. The profile only depends on the depth in the ice, causing rays still to be restricted to a vertical plane as previously discussed. At the critical depth, the two exponential function are connected to each other resulting in a non-differentiable continuity in the model. As an example, the firn model for Summit Station [2] is given below.

$$
\begin{aligned}
& n(z)=1.78-0.502 \cdot \exp (-z / 30.8 \mathrm{~m}) \\
& z \leq 14.9 \mathrm{~m} \\
& n(z)=1.78-0.310 \cdot \exp (-(z-14.9 \mathrm{~m}) / 40.9 \mathrm{~m}) \\
& z \geq 14.9 \mathrm{~m}
\end{aligned}
$$

\subsection{Horizontal propagation}

Horizontal propagation is introduced as a solution for signals originating from the classically forbidden region called the shadow zone, which can be accommodated in the RadioPropa module. The ray bending deducted from the exponential profile and Fermat's least-time principle, dictates the existence of these forbidden zones for signals. However, the ARIANNA experiment, has seen small signals coming from these regions [3], implying the existence of new modes for electromagnetic waves such as horizontal waves. An adapted module, called HorizontalPerturbation, makes it 

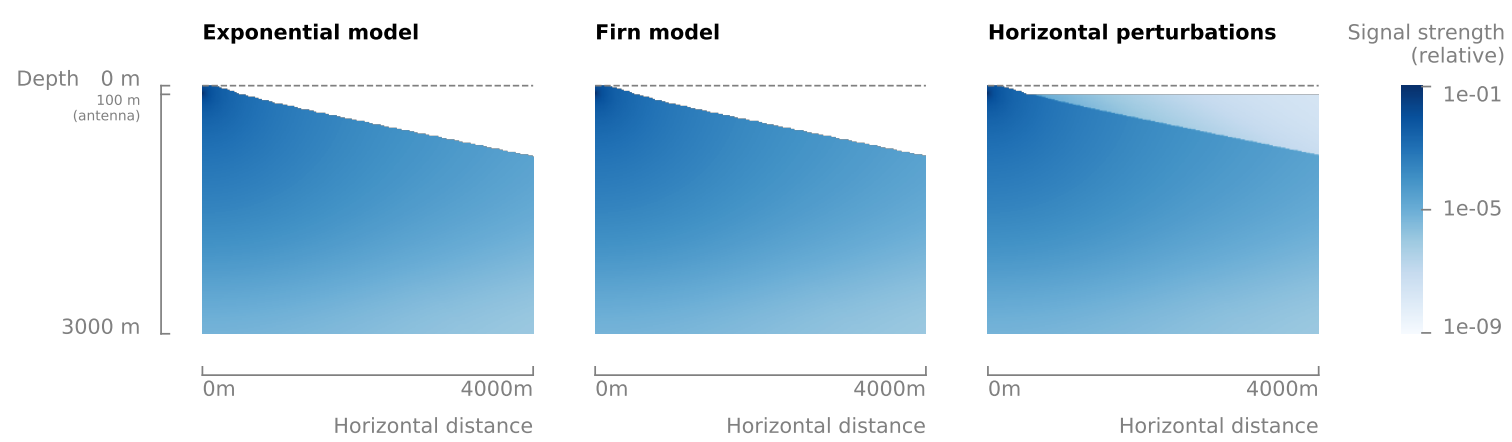

Figure 4: For Summit-Greenland, the firn model has a negligible influence on the shadow zone (white) compared to the exponential model for an antenna at $100 \mathrm{~m}$ depth, unlike the horizontal perturbations. The last one enlarges the detection volume upwards although the signal strength is 100 to 1000 times smaller.

possible to include these new wave modes in predefined layers. It will propagate a fraction of the signal further in the horizontal layer instead of pushing it downwards again. For Summit Station, a horizontal propagation layer with an electric field fraction of $2.3 \%$ and an attenuation length of half that of the bulk ice [2] is implemented in NuRadioMC at $100 \mathrm{~m}$ depth to investigate the effect on the shadow zone for a $100 \mathrm{~m}$ deep detector antenna. Here, the new propagation mode increases the observable area - thus decreasing the shadow zone - but the observed signal strength is low compared to the normal volume (Figure 4) and depends on the specifications of the new layer.

\section{Implications of the firn model for neutrino simulations}

Swapping out the analytic ray tracer for the RadioPropa makes it possible to experiment with new and more complex ice models and investigate their influence on the signal detection. As example, a neutrino simulation using the firn model is compared to a simulation depending on the exponential model. This simple comparison indicates already non-trivial changes in the detected signals.

The difference in refractive index between the firn and exponential model goes up to only $2.1 \%$ but this significantly affects the arrival time, depending on ray type and depth. At $350 \mathrm{~m}$ deep the firn model starts to differ from the exponential model resulting in a maximal difference at $14.9 \mathrm{~m}$ deep, not surprisingly the critical depth of the model (Figure 5 left). Investigating detector antennas at depths of $100 \mathrm{~m}, 50 \mathrm{~m}$ and $20 \mathrm{~m}$, this firn model introduces a non-trivial time shift depending on both the depth of the detector and the ray type (Figure 5 right). An antenna closer to the surface predominantly experiences signals arriving later, while for a deeper antenna the direct rays shift more to earlier times, unlike reflected rays. That is because the speed of light in the firn model is faster between depths of $50 \mathrm{~m}$ to $350 \mathrm{~m}$, and slower near the surface, compared to the exponential model. This affects direct rays the most as they do not spend an equal amount of time in the upper layers for antennas at different depths. For antennas at large depths, some refracted rays traverse shallow depths and others do not, resulting in a spread across both early and late times, unlike for near-surface antennas. 

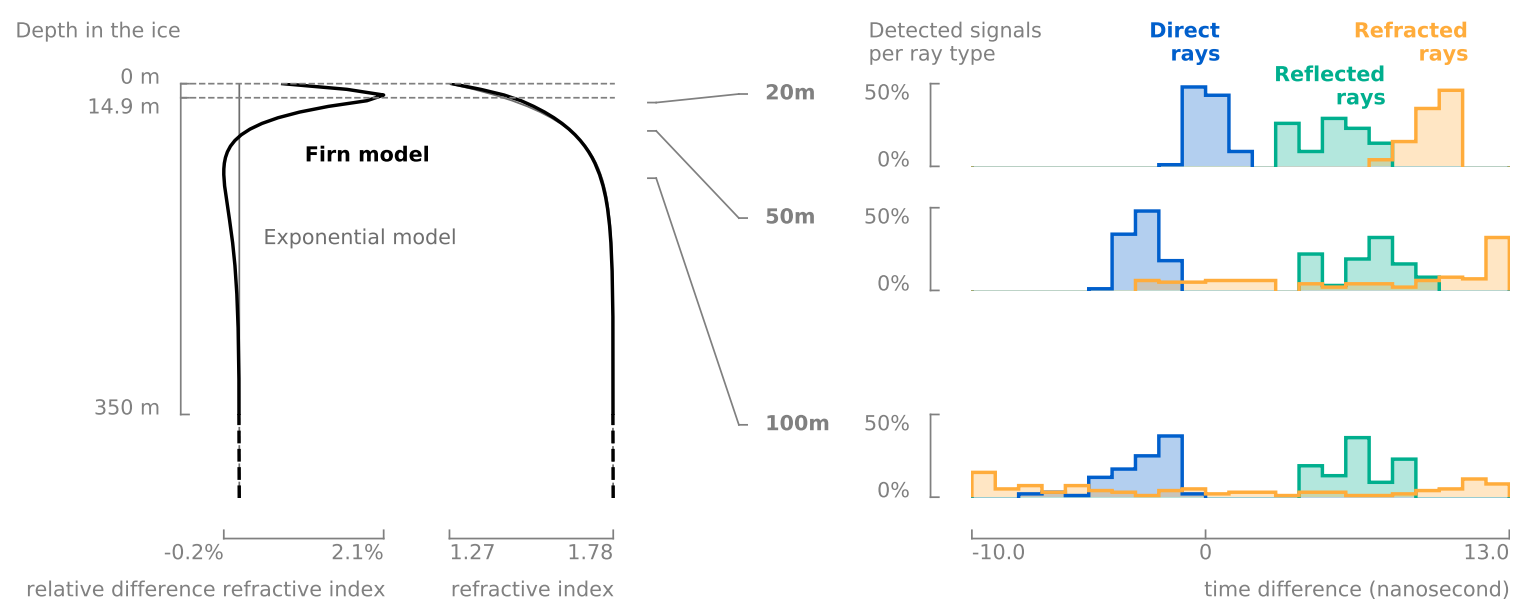

Figure 5: The difference in refractive index between the firn model and the exponential model (left) is confined to the upper $350 \mathrm{~m}$ of the ice and limited to a deviation of $2.1 \%$ from the exponential model with the largest difference located at the non-differentiable point of the firn model. The resulting difference in signal arrival time, evaluated here at $20 \mathrm{~m}, 50 \mathrm{~m}$ and $100 \mathrm{~m}$, depends on both ray type and depth of the detector's antenna (right). In the firn model, direct rays arrive predominantly earlier and reflected rays always arrive later, and this difference becomes more prominent for deeper antennas.

A first simulation study using a RNO-G-like [1] station indicates that both angular resolution and distance resolution are effected by the change in model and the resulting time shifts. The time delays between antennas of the detector are used to reconstruct the signal arrival direction, whereas time differences between different ray types are used to determine the vertex distance. Therefore, the reconstruction is affected by changes in timing. Neutrino events without thermal noise of the detector were simulated for both the firn model and exponential model, and afterwards reconstructed utilizing the exponential ice model [6]. To evaluate the implications we compare the differences of the reconstructed values to the simulated values. The angular resolution on the signal's zenith obtained a larger and asymmetric spread, shifting the zenith towards higher values (Figure 6). The resolution of the neutrino vertex distance shows a similar behaviour but with a symmetric spread. The resolution on the signal's azimuth remains the same as the firn model has no lateral component.
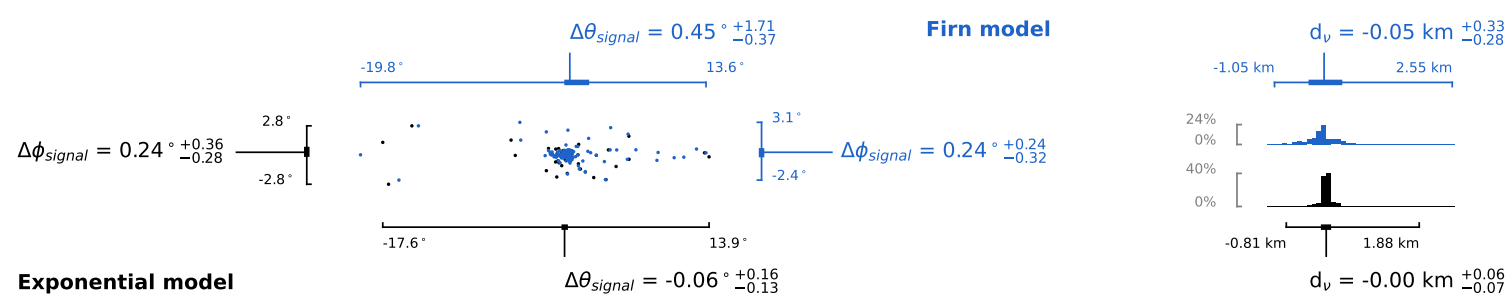

Figure 6: The azimuthal resolution of the signal direction barely changes, whereas the zenithal resolution and the vertex distance shift to higher values with a larger spread. The resolution is characterised by the mode (most probable value) with a $68 \%$ interval on both sides of the mode. 


\section{Conclusion \& Outlook}

The new developed RadioPropa ray tracing module in NuRadioMC facilitates the use of nonexponential ice models so the simulations and reconstructions can be optimised. More complex ice models can describe better what is expected from real glacial ice, but up to now the ray tracing for such models could not be solved in NuRadioMC. The RadioPropa module changes this by adding a numerical raytracer. Currently RadioPropa takes up to 50 times longer compared to the analytic ray tracer. However, a faster minimization method is being developed, besides the currently implemented iterative method. Because the ray tracer is numerical, any three-dimensional scalar refractive index models can be used, bearing in mind that specific features may require additional modules such as horizontal propagation. At the moment, RadioPropa is set up for depth dependent ice models only. Nevertheless, the double-exponential firn model already introduces a non-trivial time shift for the arrival signal, depending on both the depth of the antennas of the detector and the ray type of the signal. These differences in timing influence both the reconstruction of the distance to the neutrino vertex and the reconstruction of the zenith of the signal direction. Therefor, reconstruction methods will be optimised to take into account new ice models and their uncertainties. The quest for adequate ice models is still ongoing, where even the complex ice models are still far more simpler than the real ice, thus not all ice effects are currently accounted for. Additional important ice features to include are internal reflective layers and birefringence, the latter being the effect of having a different refractive index for different components of the electric-field and different directions in the ice. However, as birefringence is a three-dimensional effect, the azimuthal restriction on the scanning method will need to be revisited. In the future we plan to include more of such complex ice features providing more accurate and realistic simulations, which will be used to develop improved reconstruction methods.

\section{References}

[1] J. Aguilar et al., Design and sensitivity of the Radio Neutrino Observatory in Greenland (RNO-G), Journal of Instrumentation 16 (2021) P03025.

[2] C. Deaconu et al., Measurements and modeling of near-surface radio propagation in glacial ice and implications for neutrino experiments, Physical Review D 98 (2018) .

[3] S. Barwick et al., Observation of classically 'forbidden' electromagnetic wave propagation and implications for neutrino detection., Journal of Cosmology and Astroparticle Physics 2018 (2018) 055-055.

[4] C. Glaser et al., NuRadioMC: simulating the radio emission of neutrinos from interaction to detector, The European Physical Journal C 80 (2020).

[5] T. Winchen, RadioPropa - A Modular Raytracer for In-Matter Radio Propagation, EPJ Web of Conferences 216 (2019) 03002.

[6] I. Plaisier for the RNO-G Collaboration, Direction Reconstruction for the Radio Neutrino Observatory Greenland (RNO-G), Proceedings of Science - $37^{\text {th }}$ International Cosmic Ray Conference (2021) . 